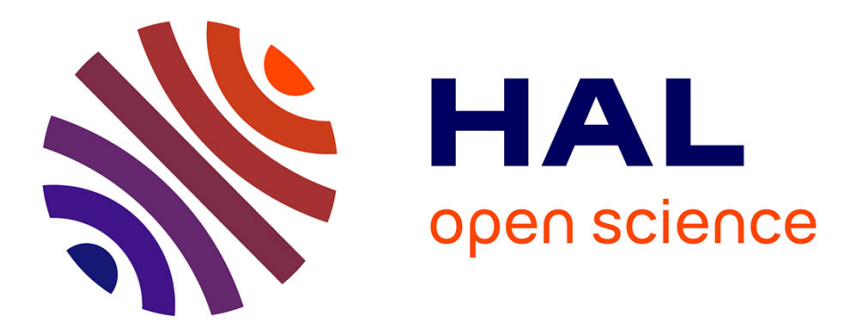

\title{
CT-scan images preprocessing and segmentation to improve bioprosthesis leaflets morphological analysis.
}

Vito Giovanni Ruggieri, Pascal Haigron, Qian Wang, Simon Esneault, Raphael Madeleine, Jean-François Heautot, Alain Leguerrier, Jean-Philippe Verhoye

\section{- To cite this version:}

Vito Giovanni Ruggieri, Pascal Haigron, Qian Wang, Simon Esneault, Raphael Madeleine, et al.. CTscan images preprocessing and segmentation to improve bioprosthesis leaflets morphological analysis.. Medical Hypotheses, 2013, 81 (1), pp.86-93. 10.1016/j.mehy.2013.03.032 . inserm-00821092

\section{HAL Id: inserm-00821092 https://www.hal.inserm.fr/inserm-00821092}

Submitted on 7 May 2013

HAL is a multi-disciplinary open access archive for the deposit and dissemination of scientific research documents, whether they are published or not. The documents may come from teaching and research institutions in France or abroad, or from public or private research centers.
L'archive ouverte pluridisciplinaire HAL, est destinée au dépôt et à la diffusion de documents scientifiques de niveau recherche, publiés ou non, émanant des établissements d'enseignement et de recherche français ou étrangers, des laboratoires publics ou privés. 
CT-scan images preprocessing and segmentation to improve bioprosthesis leaflets morphological analysis

V.G. Ruggieri, MD, PhD, P. Haigron, PhD, Q. Wang, S. Esneault, PhD, R. Madeleine, JF Heautot, $\mathrm{PhD}$, A. Leguerrier, J-Ph. Verhoye, $\mathrm{PhD}$.

CHU Rennes, Department of Cardiovascular and Thoracic Surgery, Rennes, France INSERM, U1099, Rennes, France

Université de Rennes 1, LTSI, Rennes, France

Address for correspondence:

Vito Giovanni RUGGIERI, MD, PhD

Service de Chirurgie Thoracique et Cardiovasculaire

Hopital Pontchaillou

2, rue Henri Le Guilloux

35003 Rennes Cedex 9, France

Tel\# 0033-299-28-91-64

Fax\# 0033-299-28-24-96 


\begin{abstract}
The visualization of bioprosthesis leaflet morphology might help to better understand the underlying mechanism of dysfunction in degenerated aortic bioprosthesis. Because today such visualization of bioprosthesis leaflet morphology is intricate to impossible with other imaging techniques, we hypothesized that the processing of multi-detector CT images would allow better visualization of the prosthetic valve leaflets after biological aortic valve replacement. The purpose of our study was to prospectively evaluate patients with a degenerated aortic bioprosthesis, waiting for reoperation, by using 64-slice CT to evaluate prosthetic leaflets morphology. A semi-automatic segmentation of pre-operative tomodensitometric images was conducted, using 2 different implementations of the region growing algorithm. Here we report all segmentation steps (selection of the region of interest, filtering, segmentation). Studied degenerated aortic bioprostheses were represented by two Carpentier-Edwards Supra Annular Valve (porcine leaflets), one Edwards Perimount (pericardial leaflets) and one Medtronic Mosaic (porcine leaflets). Both segmentation methods (Isotropic Region Growing and Stick Region Growing) allowed a semi-automatic segmentation with 3D reconstruction of all bioprosthetic components (stent, leaflets, degeneration/calcifications). Explanted bioprosthesis $\mathrm{CT}$ images were also processed and used as reference. Segmentation results were compared by means of quantitative criteria. Semi-automatic segmentation using region growing algorithm seems to provide an interesting approach for the morphological characterization of degenerated aortic bioprostheses. We believe that in the next future CT scan images segmentation may play an important role to better understand the mechanism of dysfunction in failing aortic bioprostheses. Moreover, bioprostheses 3D reconstructions could be integrated into preoperative planning tools to optimize valve-in-valve procedure.
\end{abstract}




\section{INTRODUCTION}

During the last decade, the relative use of bioprosthetic aortic valves has clearly increased. Improvements in surgical techniques and valve durability are likely to have fueled this increase. On the other hand, the mortality risk of redo aortic valve surgery has similarly decreased. Together with the best postoperative quality of life, these good surgical results contribute to the large diffusion of bioprostheses implantation even in younger patients. This phenomenon, associated to the increased life expectancy in industrialized countries, will probably show its consequences in the next years when a large number of elderly patients with associated comorbidities will need a re-intervention for bioprosthesis degeneration. With the introduction of the transcatheter aortic valve to treat native aortic valve stenosis in high or prohibitive surgical risk patients through a less invasive technique, medical community has discovered a seducing but still emergent option to treat bioprosthesis failure in the same kind of patients. Transcatheter valve implantation avoids re-sternotomy and cardiopulmonary bypass and can potentially reduce resource utilization by accelerating patient recovery and reducing hospital stay. The major failure mechanism for bioprostheses is represented by leaflet degeneration and the current reference standard for follow-up in patients after aortic valve replacement remains trans-thoracic echocardiography, with trans-esophageal exploration in case of questionable findings at trans-thoracic. Echocardiography represents the gold standard for functional assessment of bioprostheses. However, both trans-thoracic and trans-esophageal echocardiography have limited usefulness for morphological assessment, especially concerning bioprosthetic leaflets, because of their thickness and the acoustic shadowing from stent or suture ring that often prevents their good visualization. Furthermore, given their two-dimensional imaging nature, it is generally impossible to directly image the leaflets that are not oriented in the imaging plane. Even the new 3D echocardiography has, for 
the moment, limited application on the aortic bioprostheses also because of the anterior position.

During the last years, multi-detector computed tomography (CT) has shown its potential to provide precise diagnostic information in different cardiac clinical situations, such as noninvasive evaluation of coronary disease [1-6], ventricular morphology and function [7], and myocardial viability [8-10]. Multi-detector CT can also help visualize the morphology and motion of native and diseased stenotic aortic valves and precisely measure aortic valve opening areas. Several studies [11-17] suggest that multi-detector CT can also help assess mechanism of dysfunction in mechanical prosthetic heart valve disorders.

In clinical practice CT scan images normally allow a tridimensional reconstruction of the structures of interest, thanks to their high spatial resolution. The 3D analysis improves the users understanding and facilitates the planning of surgical/interventional procedures.

In this context, the starting idea of this research work was that the tridimensional reconstruction of bioprosthetic structures, in particular of prosthetic leaflets, could make easier the morphological analysis of degenerated bioprostheses during normal follow-up and be used for patients' selection, improved planning and simulation of valve-in-valve procedure in the future. Currently, the available softwares to analyze and process CT scan images do not allow an efficient and effective tridimensional reconstruction of the bioprosthetic leaflets: bovine pericardium and porcine valve leaflets are in fact too thin and CT images are also altered by noise, stent metal artifacts and heart beat. This makes difficult the automatic analysis with available tools and new methods to process these high resolution images have to be considered.

We hypothesized that multi-detector CT would allow better visualization of the prosthetic valve leaflets after biological aortic valve replacement. Accordingly, the purpose of our study 
was to prospectively evaluate patients with a degenerated aortic bioprosthesis, waiting for reoperation, by using 64-slice CT to morphologically analyze bioprosthetic leaflets.

\section{METHODS}

\section{Study Protocol}

The study protocol was approved by the institutional review board. Written informed consent was obtained from patient after they were explained about the radiation exposure. Patients with a failing aortic bioprosthesis and waiting for reoperation at our institution were included in the study. All patients were in stable hemodynamic condition. Exclusion criteria were as follows: urgent operation (hemodynamic instability or supine position suffering), constant arrhythmia (atrial fibrillation or more than five premature heart beats per minute), New York Heart Association class IV heart failure, renal insufficiency (serum creatinine level greater than $1.4 \mathrm{mg} / \mathrm{dL}$ ), and known allergy to iodinated contrast agents.

In-Vivo scanning protocol (Coronary CT protocol) : in order to reduce motion artifacts, included patients underwent preoperative electrocardiographically gated cardiac multidetector CT performed with a 64-slices system (General Electric Medical Systems VCT 64 or Discovery 750HD, GE Healthcare, Waukesha, WI). Patients with a heart rate of more than 75 beats per minute received oral Bisoprolol $5 \mathrm{mg}$ /day for 2 days prior to CT associated to intravenous Atenolol (intravenous bolus injection of $5 \mathrm{mg}$ before exam) if they had no contraindication to beta-blocker administration.

The scanning direction was craniocaudal and extended from the level of the carina to the diaphragm. Prior to scanning, a technologist instructed all patients regarding breath holding in an effort to minimize changes in body posture during the examination. The scanning sequence was as follows: topography was performed and was followed by a bolus tracking examination and two coronary CT angiography algorithms. 
The intravenous bolus of contrast medium was tracked in the ascending aorta at the level of the pulmonary trunk every 2 seconds. The administered contrast medium was Iobitridol (Xenetix) (Guerbet Aulnay-sous-Bois, France) or Iohexol (Omnipaque) (GE Healthscare Healthcare, Inc. Princeton, NJ). The intravenous triple bolus was realized with $95 \mathrm{ml}$ of contrast medium, followed by $45 \mathrm{ml}$ of a mix of contrast and saline solution $(50 / 50 \%)$ and finally rinsed by $30 \mathrm{ml}$ of saline solution at $5 \mathrm{ml} / \mathrm{sec}$. Individual body weight-adapted volume of contrast media and injection rate were not performed.

Retrospective CT angiography was performed with the following parameters: helical scanning direction, 233-msec x-ray exposure time (two-thirds of the gantry rotation speed), 64 x 0.625 mm collimation, 0.35 -second gantry rotation time, $100-\mathrm{kV}$ tube voltage, $0.16-0.22$ pitch and use of dose modulation (peak tube current of $750 \mathrm{~mA}$ during $0 \%-90 \%$ of the R-R interval and minimal tube current of $300 \mathrm{~mA}$ ) to reduce DLP (Dose Length Product in mGy x cm).

Retrospective reconstruction of multiple phases of cardiac cycle was performed. The diastole phase ( $70 \%$ of cardiac cycle) was considered as the best to study the aortic bioprosthesis with leaflets in their closed position.

Ex-Vivo scanning protocol (Temporal bone protocol) : after reoperation and intraoperative evaluation of the failure mechanism, CT-scan images of the isolated explanted aortic bioprosthesis were obtained using a high image resolution protocol with the following parameters: 64 x $0.3125-\mathrm{mm}$ collimation, 1-second gantry rotation time, $140-\mathrm{kV}$ tube voltage, 0.531 pitch and tube current of $170 \mathrm{~mA}$.

\section{Patient Population}

During a 2 years period, 9 patients were considered for the study. Among these, only 4 patients were included according to selection criteria. Three patients were excluded because of associated stable arrhythmia, heart failure (requiring emergency care) and renal 
insufficiency which contraindicated the use of iodinated contrast enhanced imaging. Degenerated bioprostheses were represented by two Carpentier-Edwards Supra Annular Valve (Case 2 - MAG and 3 - BOU), one $23 \mathrm{~mm}$ Edwards Perimount (Case 1 - HER) and one $23 \mathrm{~mm}$ Medtronic Mosaic (Case 4-DAV). Bioprostheses echocardiographic characteristics and failure mechanisms are shown in table 1.

\section{Image Processing}

A semi-automatic segmentation of pre-operative tomodensitometric images was conducted, using 2 different implementations of the region growing algorithm. Here we report all segmentation steps.

\section{1 - Selection of the region of interest}

In order to deal with the difficulties of segmentation of the bioprosthesis CT images (metal artifacts, thickness of leaflets compared with image resolution, density of valve components compared to stent) we considered a region of interest (ROI) defined by the stent. According to the type of bioprosthesis stent implanted in the patient, we considered two kind of shape for the ROI: a cylindrical shape preferentially for incomplete metallic stents and a conformational shape for complete metallic stents (Fig. 1).

\section{2 - Preprocessing}

A preprocessing step is implemented in order to reduce the noise in CT images. The goal is to increase the signal to noise ratio (SNR) as well as to increase the contrast of the structures of interest, especially the valve leaflets. We considered methods based on diffusion filters (curvature and anisotropic diffusion filter) as well as an adaptation of stick filters, initially introduced to segment ultrasound images, and to reduce noise and keep details in thin structures $[18,19]$. To select the most appropriate approach before the segmentation step, we 
considered the SNR as the performance criterion. To compute the SNR we used the definition given in [20]:

$$
S N R=10 * \log _{10}\left(\frac{\operatorname{Max}\left(\operatorname{Var}_{i}\right)}{\operatorname{Min}\left(\operatorname{Var}_{i}\right)}\right)
$$

where $\operatorname{Var}_{i}$ is the the local variance in the neighborhood of leaflets and vascular lumen voxels located in the ROI. Voxels located at the boundary between leaflets and vascular lumen are used to obtain the maximum variance whereas voxels located in the vascular lumen are used to obtain minimum variance. Due to their high value, voxels representing stent, calcifications and pannus are not considered in the computation of SNR.

The diffusion filters are non-linear filters based on partial differential equations. They have been proposed for noise reduction and segmentation, particularly for vascular images.

In classical Perona-Malik (PM) Model [21], the values of resulting voxels $I_{p}^{t+1}$ are calculated as:

$$
I_{p}^{t+1}=I_{p}^{t}+\frac{\lambda}{\left|\eta_{p}\right|} \sum_{q \in \eta_{p}} c\left(\nabla I_{p, q}^{t}\right) \nabla I_{p, q}^{t}
$$

where $I_{p}^{t}$ is the current value of the voxel. $\nabla I_{p, q}^{t}$ is the discrete gradient along the $\mathrm{q}^{\text {th }}$ direction (defined by the current voxel and a voxel of the neighborhood). $c(x)$ is the classifier between edge and noise. $c(x)$ can take different expressions such as:

$$
c(x)=\frac{1}{1+(x / k)^{2}} \text { or } c(x)=\exp \left(-(x / k)^{2}\right),
$$

where $\eta_{p}$ is the neighborhood of the current voxel and $\left|\eta_{p}\right|$ is the number of voxels in $\eta_{p} . \lambda$ and $k$ are two parameters to control diffusion intensity. 
Classical Anisotropic diffusion operators as PM model can enhance edge while denoising the image. Nevertheless, they may exhibit both edge and noise in low SNR conditions. Anisotropic Curvature Diffusion, which is based on a modified curvature diffusion equation (MCDE) [22-24], has been introduced to overcome this problem and to be less sensitive to noise.

In Anisotropic Curvature Diffusion, the Discrete Gradient $\nabla I_{p, q}^{t}$ used in PM Model was replaced by Gaussian Curvature of the voxel:

$$
I_{p}^{t+1}=I_{p}^{t}-\frac{\lambda}{N} \sum_{q \in \eta_{p}} c\left(\operatorname{Curv}\left(P_{p, q}^{t}\right)\right) \operatorname{Curv}\left(P_{p, q}^{t}\right)
$$

where $P_{p, q}^{t}$ is the plane defined by the current voxel and a direction given by a voxel of the neighborhood $\eta_{p} \cdot \operatorname{Curv}\left(P_{p, q}^{t}\right)$ is the Gaussian Curvature at the voxel.

As suggested by Perona et al [21], in the proposed CT data pre-processing approach we chose $\left|\eta_{p}\right|=26$ and $\mathrm{N}=9\left(3 * 3 * 3\right.$ cubic neighborhood). Higher values of $\eta_{p}$ may smooth edge and increase computational complexity too much. $c(x)=\frac{1}{1+(x / k)^{2}}$ was selected thanks to its simple computational complexity. Values of $\lambda$ and $\mathrm{k}$ were set at 1 for a low level of noise (high $\mathrm{SNR}$ ). In the case of images showing a high level of noise $(\mathrm{SNR}<=8), \lambda$ and $\mathrm{k}$ were set at 0.5. In general, $\mathrm{K}$ value should be set in a range of grey level values between the gradient leaflet/vascular lumen and the gradient inside the noise.

Stick filter is also a non-linear filter. With stick filtering approach, the neighborhood of the considered voxel is divided into a set of asymmetric sticks to perform a non-linear filtering of the image. Because of its asymmetric stick, the filter could get better performance in terms of keeping details of thin structure, as valve leaflets, while denoising the image. 
The filter output I' at the current voxel was originally defined as:

$$
I^{\prime}=\frac{1}{W} \sum_{i=1}^{N} g_{i} * \bar{I}_{i} \text { and } W=\sum_{i=1}^{N} g_{i}
$$

where $\bar{I}_{i}$ denotes the local mean value along the $i^{\text {th }}$ stick, $N$ is the number of sticks, $g_{i}$ is the reciprocal of local variance along the $i^{\text {th }}$ stick, used to classify edge and noise.

This filter was adapted to pre-process CT images of degenerative aortic valve bioprosthesis. In our approach, the resulting value of the filtering process was considered as the sum of voxel median values normalized by the standard deviation along 4 voxels length sticks defined in the neighborhood. The values of resulting voxels $I^{\prime}$ were more precisely defined as:

$$
I^{\prime}=\frac{1}{W} \sum_{i=1}^{N} g_{i} * I_{M i} \text { and } W=\sum_{i=1}^{N} g_{i}
$$

where $I_{M i}$ denotes the local median value along the $i^{\text {th }}$ stick, $N$ is the number of sticks ( $N=24 L^{2}-48 L+26$ in 3D conditions), $L$ is the number of voxels along the stick, and $g_{i}$ is the reciprocal of local variance along the $i^{\text {th }}$ stick.

$L$, the length of stick, is the main parameter of this filter. We tested different values of $\mathrm{L}$, from 3 to 7 . L should be larger than texture caused by noise and smaller than thin structure (leaflet). We found that normally, $\mathrm{L}=5$ is suitable for our data sets (voxel is $0.312 * 0.312 * 0.625 \mathrm{~mm}^{\wedge} 3$, thickness of leaflets is $0.5-1 \mathrm{~mm}$, width of noise texture is different in data sets, $3-5$ voxels). Other values of $\mathrm{L}$ can be used if the parameters of data set change. $\mathrm{L}$ is also determined by SNR of original CT images. When $\mathrm{SNR}<=8, L=5 ; 8<\mathrm{SNR}<=10, L=4 ; \mathrm{SNR}>10, L=3$.

Besides, the number of iteration is not a critical issue unlike Diffusion Filter. Stick Filter shows a convergent behavior after several iterations. Typically, in a low SNR condition $(\mathrm{SNR}<=8)$, the result converged after 5 iterations (after 3 iterations when $\mathrm{SNR}>10$ ). It was not necessary to limit the number of iterations to avoid an over-smoothing of edges. 
The drawback of Stick Filter is its high computation complexity. A 5 voxels-length Stick Filter (i.e. a $9 * 9 * 9$ cubic neighborhood), requires for each voxel and at each iteration, the calculation of 386 local medians and local variances. The time of calculation can be decreased by the stent-defined ROI. The number of voxels located in the ROI is much less than in the cubic volume encompassing the aortic valve bioprosthesis. Cylindrical ROI typically decreases the number of voxels to $40 \%$ and conformational ROI to $16 \%$.

\section{3 - Valve segmentation}

According to surgical expertise, four main classes of tissues were identified. They were related to the Hounsfield Units (HU) measured by CT. We considered, from high to low HU, the following classes: stent, calcifications, vascular lumen and leaflets. The first two elements, showing high CT values, can be easily segmented by thresholding; the issue of segmentation is more particularly focused on bioprosthetic leaflets.

Two kinds of region-based segmentation processes were implemented, with a decreasing level of user interactivity.

\section{A - $\quad$ Isotropic Region Growing (IRG)}

It was applied using the 3D Slicer software, an open-source application that assists with the visualization, registration, segmentation and quantification of medical image data. It can be downloaded at http://www.slicer.org.

Multiple seed points were interactively selected for each component of the prosthesis. The region growing algorithm was started (26-neighbour connectivity) with automatic calculation of the criterion used for the aggregation of voxels. This similarity criterion was based on mean and standard deviation of the voxel values within the considered cubic neighborhood [25]. The extraction and visualization of the resulting 3D surface meshes delineating the regions were performed by using the Marching Cube algorithm [26]. 


\section{B - $\quad$ Stick Region Growing (SRG)}

To better deal with the segmentation of thin structures, like the components of a valve bioprosthesis and especially valve leaflets, we developed a directional region growing process based on stick neighborhood. The segmentation was thus performed by the stick region growing algorithm applied to a previously selected region of interest (ROI).

Some seeds were interactively selected inside the leaflets at different locations (typically 4 to 6 seeds). A set of $\mathrm{N}$ voxels inside the leaflets was defined by considering for each seed the voxels belonging to their neighborhood. The similarity criterion was locally based on mean and standard deviation of the CT voxel values along the directions defined by the sticks. This approach was implemented using MatLab development tools.

In order to examine each voxel in the cubic neighborhood of the seed, we specifically computed the stick variances in the set of sticks defined by each of these voxels:

$$
\operatorname{Var}=\frac{\sum_{j=1}^{L}\left(I_{t, j}-\bar{T}_{i}\right)^{2}}{L}, \quad \text { where } L \text { is the length of the stick. }
$$

$\mathrm{L}$ was chosen to be less than the thickness of the leaflets. Typically $\mathrm{L}$ was between 3 and 5 voxels (about 1mm).

To ensure region growth is not early stopped, or in other words to limit the number of seed points selection in some critical cases (highly noisy data), the size of the cubic neighborhood was chosen to be large enough to include space between homogeneous fragment of leaflets. We typically used a 342-connected neighborhood $(7 \times 7 \times 7)$. We also defined $\overline{I_{m}}=\frac{1}{L} \sum_{j=1}^{L} I_{m, j}$, the local mean of the stick with minimum local variance.

The voxels were classified as belonging to the region of the seed voxel (i.e. as a voxel of normal leaflet tissue) when: (i) $\overline{I_{m}}$ belongs to the grey level range of normal leaflets and (ii) minimum local variance along stick is lower than a variance threshold (VTH). VTH was 
determined by the difference of local cubic neighborhood variances of leaflets voxels and boundary voxels. These variances have been previously calculated in SNR computation (for image preprocessing). When this voxel belonged to the ROI, it constituted a new seed voxel. This process was iterated until no new voxel can be marked as a seed.

\section{RESULTS}

We obtained preoperative CT images (in-vivo) in four patients (HER, MAG, BOU, DAV) presenting a degeneration of aortic bioprosthesis. The radiation dose for each patient was comprised between 440 and 480 DLP (Dose Length Product - mGy x cm). Depending on the case, the reoperation was performed from 7 to 19 years after implantation for different pathological reasons. In one case it was a pericardial bioprosthesis (HER), in which bovine pericardium is cut to reproduce aortic valve leaflets that are sewn on a complete metallic stent. Three more cases were represented by porcine bioprostheses, in which a porcine aortic valve is mounted on a complete metallic stent ( 2 cases: MAG and BOU) or incomplete metallic stent (1 case: DAV). After reoperation, CT-scan images of isolated explanted aortic bioprosthesis were obtained using the highest image resolution protocol (ex-vivo). Table 2 shows the results of resolution parameters for in-vivo and ex-vivo images.

The preprocessing based either on diffusion or on stick filter was applied to the in-vivo CT volumes of the four patients. The bioprosthesis voxels were first isolated by considering the ROI defined by the stent. An example of original image and the result of two different filters is reported in Fig. 2. A first qualitative appreciation of the results let appear that stick filter based approach better enhance the contrast between valve leaflets and surrounding structures.

In addition to this qualitative assessment, the SNR was computed on original images and resulting images (Fig. 3). This objective assessment showed that stick filtering had the best SNR in the four patients. This was consistent with the subjective appreciation. 
Following the ad hoc preprocessing step, the different segmentation methods (IRG and SRG) were applied to the in-vivo (IV) and ex-vivo (EV) images. Ex-vivo images, easier to segment with both methods and showing similar results, were used as reference.

Figures 4 and 5 show some examples of segmentation with both methods. The first case of a segmented pericardial bioprosthesis (HER) showed minimal leaflet degeneration. The leaflet prolapse resulting in imperfect coaptation appears as commissure lost in the segmented images. The second case (MAG), a porcine bioprothesis, was characterized by a calcified pannus that was responsible for valve obstruction. The pannus was not detected at preoperative echocardiography.

In the third case (BOU) the calcification of a leaflet was responsible for a functional stenosis of the porcine bioprosthesis. The last case (DAV) was represented by a porcine bioprosthesis with an incomplete metallic stent. This bioprosthesis, considered as normal at preoperative echocardiography, was explanted during a surgery for infected ascending aorta pseudo-aneurysm. Intra-operative evaluation showed leaflets thickening as starting process of infective endocarditis.

A quantitative analysis was then conducted to objectively compare the results obtained respectively by IRG and SRG on in-vivo (IV) and ex-vivo (EV) images. The criterion used to evaluate the results was the volume of bioprothesis components computing the voxel volume and counting the number of voxels for each component (stent, leaflets, and calcifications). Results of this analysis are shown in Fig. 6 (volumes are expressed in $\mathrm{mm}^{3}$ ). When we look at stent results we find that the stent of the first case is bigger in volumes comparing to the others. This corresponds to reality because the stent of the bioprosthesis Edwards Perimount is volumetrically bigger than the Carpentier-Edwards Supra Annular Valve or the Medtronic Mosaic stents. Moreover stent volumes in vivo are bigger than ex-vivo. Concerning leaflets, volumes results show a good coherence between methods. Case 4 - DAV is characterized by 
an incomplete stent. In this case segmentation considered the basal part of the non metallic stent as leaflet. This explains the big volumes of leaflets in this case. The studied bioprostheses doesn't show leaflets strongly calcified. Only the case 2 - MAG was characterized by a calcified sub-valvular pannus that was well represented in volume analysis.

\section{DISCUSSION}

An aortic bioprosthesis can have functional degradation and prosthesis dysfunction over time $[27,28]$. In more recent bioprostheses, the rate of structural failure appears to be somewhat lower $[29,30]$ than with the first generation, but it is still occurring.

Obstruction in aortic bioprosthesis may be caused by the presence of thrombus on the leaflets or stents, by fibrous or calcified pannus (ie, fibrous tissue growing within the bioprosthesis due to excessive cicatrisation) or by the structural deterioration of leaflets due to calcification. Prosthetic thrombosis may be suspected clinically when the bioprosthesis shows acute deterioration of its effective orifice area (EOA). Fibrous pannus and structural deterioration may be suspected when EAO decreases slowly over time. Treatment is different: thrombosis may reverse with anticoagulation treatment. Structural deterioration and fibrous pannus are irreversible and typically require reoperation with replacement of the bioprosthesis, which has a high risk of surgical morbidity and mortality, especially in elderly patients with associated comorbidities. Moreover, in the next future, these high risk patients suffering from a structural valve deterioration of their bioprosthesis, could benefit from the new techniques of transcatheter aortic valve implantation (valve-in-valve procedure) [31].

Understanding the precise mechanism of bioprosthesis dysfunction would thus be important to direct clinical management. Yet, it can often be difficult to identify these entities in clinical practice. Indeed, both transthoracic and transesophageal echocardiography have limited usefulness for detection of the mechanism of stenotic bioprostheses, because of the acoustic shadowing from valve stents or annulus. 
CT images would potentially allow better visualization of the bioprosthetic valve leaflets after aortic valve replacement, but at the moment CT scan softwares don't allow automatic leaflets enhancement. In this study we have tested two different implementations of the region growing segmentation on CT images of degenerated aortic bioprosthesis. These two methods, considered as semiautomatic segmentation methods, seem to be similar in terms of results even if different in their approach.

The pre-processing represents a key point of the analysis: bioprostheses CT images need to be treated before to be segmented because of noise and metallic stent artifacts.

The ROI selection reduces most of the effects of the artifacts. In fact we define a ROI in order to stop region growing into artifacts. Normally, metal artifact reduction needs a process of radon translation, interpolation and in-translation. These represent very complex methods that often loose images details as thin structure. Remaining artifacts could impact on qualitative and quantitative results of segmentation.

Different filters have been tested in order to improve images by noise reduction and to reach the best leaflets enhancement. Stick filtering showed the best results and this was confirmed by SNR analyses. The stick filtering process can directly reduce metal artifacts (without the need for radon translation) keeping a reliable surrounding tissue. This filtering process is performed by MatLab software and it takes time to be finalized. To reduce this computing time it is important to select a ROI that takes into account only the bioprosthesis stent region with its leaflets. Stick processing time is estimated in 2 hours for each data set from original images to segmentation. The algorithm can be optimized using more processors to parallelize the process and obtain faster results in order to improve its clinical application.

Concerning segmentations results, we have some considerations. Among the analyzed data sets, the best results have been obtained in the case of the pericardial bioprosthesis. The reason 
has to be attributed to the thickness of the valve tissue: pericardial leaflets are largely thicker than porcine with consequent better detection by CT scan.

There are different factors that could explain the volumes differences observed in some cases for in-vivo images comparing to ex-vivo. Firstly, image resolution is not the same. In-vivo acquisition protocol represents the standard protocol used for coronary ECG gated angio-CTscan. Changing the acquisition parameters was not considered and specific acquisition for bioprosthesis analysis doesn't exist at the moment. For the ex-vivo images we chose the highest resolution protocol to be close to reality and to use these images as reference. Other than image resolution, we can consider heart motion and blood superposition as important factor for volumes differences. These two factors participate to modify the experimental condition of in-vivo images that explain the differences with ex-vivo images in qualitative and quantitative analysis.

Computing of leaflets volumes can be altered by the non-metallic part of bioprosthesis stent. Only the metallic stent can be used as spatial limitation for the leaflets because the non metallic part has the same density of the leaflets. For this reason leaflet volumes are higher than normal in the only case with incomplete metallic stent.

Another factor that plays a fundamental role for the quality of in-vivo images (preoperative images) is the heart rate during the exam. It should be as low as possible and not more than 70 bpm. Patient preoperative clinical conditions can be responsible for higher heart rate and contra-indicate medical therapy as beta-blockers to reduce it.

In all cases, we observed in-vivo underestimation of leaflets degeneration. This is only partially true. In fact, in the studied cases, leaflets are not so calcified and most part of degeneration consists in leaflets thickening. These leaflets modifications do not appear with the same density of calcifications and are difficult to be detected in in-vivo CT images. Only 
actual calcifications can be detected with consequent underestimation compared to ex-vivo images, in which smaller differences in density can be easily appreciated.

The study has a number of limitations: the small number of studied data sets and their heterogeneity do not allow data validation by statistical analysis. Moreover the quantitative analysis is conducted on in-vivo and ex-vivo data of bioprostheses in different experimental conditions which can explain the volumetric deviations.

Our study suggests that segmentation of CT images could play a role in clarifying the mechanism of bioprosthesis dysfunction. Indeed, we demonstrated that segmentation can help directly visualize leaflet morphology. Furthermore, we demonstrated the ability of these methods to identify bioprosthesis leaflet thickening, calcification as well as presence of pannus under bioprosthetic leaflets.

Our preliminary results suggest the feasibility to enhance aortic bioprosthesis leaflets by the application of the region growing segmentation to preoperative $\mathrm{CT}$ images after filtering process. Because today such morphological evaluation of bioprosthesis leaflets is intricate to impossible with other imaging techniques, we believe that in the next future CT scan images segmentation may play an important role to better understand the mechanism of dysfunction in patients with a degenerated aortic bioprosthesis during normal follow-up. After acquisition protocols adaptation and methods validations by larger series application, 3D reconstructions could be used for patient's selection, planning and simulation of the valve-in-valve procedure.

\section{ACKNOWLEDGMENTS}

The authors are grateful to ADETEC Society for the important contribution in this study.

\section{CONFLICT OF INTEREST STATEMENT}

All authors attest that there is no conflict of interest or financial/commercial interest in any of the products or procedures mentioned in the manuscript. 



\section{REFERENCES}

[1] Vanhoenacker PK, Heijenbrok-Kal MH, Van Heste R, et al. Diagnostic performance of multidetector CT angiography for assessment of coronary artery disease: metaanalysis. Radiology $2007 ; 244: 419-428$.

[2] Miller JM , Rochitte CE , Dewey M , et al. Diagnostic performance of coronary angiography by 64-row CT . N Engl J Med 2008 ; 359 : 2324 - 2336.

[3] Sun Z, Jiang W . Diagnostic value of multislice computed tomography angiography in coronary artery disease: a meta-analysis. Eur J Radiol 2006 ; 60 : 279 - 286.

[4] Hausleiter J , Meyer T , Hadamitzky M , et al. Non-invasive coronary computed tomographic angiography for patients with suspected coronary artery disease: the Coronary Angiography by Computed Tomography with the Use of a Submillimeter resolution (CACTUS) trial . Eur Heart J 2007 ; 28 : 3034 - 3041.

[5] Budoff MJ, Dowe D, Jollis JG, et al. Diagnostic performance of 64-multidetector row coronary computed tomographic angiography for evaluation of coronary artery stenosis in individuals without known coronary artery disease: results from the prospective multicenter ACCURACY (Assessment by Coronary Computed Tomographic Angiography of Individuals Undergoing Invasive Coronary Angiography) trial . J Am Coll Cardiol $2008 ; 52: 1724-1732$.

[6] Mowatt G, Cook JA, Hillis GS, et al . 64-slice computed tomography angiography in the diagnosis and assessment of coronary artery disease: systematic review and metaanalysis. Heart $2008 ; 94: 1386-1393$.

[7] Juergens KU, Fischbach R . Left ventricular function studied with MDCT . Eur Radiol $2006 ; 16: 342-357$. 
[8] Mahnken AH, Koos R, Katoh M, et al. Assessment of myocardial viability in reperfused acute myocardial infarction using 16-slice computed tomography in comparison to magnetic resonance imaging. J Am Coll Cardiol 2005 ; 45 : 2042 - 2047.

[9] Gerber BL, Belge B , Legros GJ , et al. Characterization of acute and chronic myocardial infarcts by multidetector computed tomography: comparison with contrastenhanced magnetic resonance . Circulation $2006 ; 113: 823-833$.

[10]Lardo AC , Cordeiro MA , Silva C , et al . Contrast-enhanced multidetector computed tomography viability imaging after myocardial infarction: characterization of myocyte death, microvascular obstruction, and chronic scar . Circulation $2006 ; 113: 394-404$.

[11] Teshima H , Aoyagi S, Hayashida N , et al. Dysfunction of an ATS valve in the aortic position: the first reported case caused by pannus formation . J Artif Organs $2005 ; 8$ : $270-273$.

[12] Teshima H , Hayashida N , Fukunaga S , et al . Usefulness of a multidetector-row computed tomography scanner for detecting pannus formation. Ann Thorac Surg 2004 ; $77: 523-526$.

[13] Teshima H , Hayashida N , Enomoto N , Aoyagi S , Okuda K , Uchida M . Detection of pannus by multidetector-row computed tomography. Ann Thorac Surg $2003 ; 75: 1631$ 1633.

[14] Aoyagi S , Fukunaga S , Arinaga K , Shojima T , Ueda T . Prosthetic valve obstruction: diagnostic usefulness of cineradiography and multidetector-row computed tomography. Thorac Cardiovasc Surg 2007 ; $55: 517$ - 519.

[15]Kim RJ , Weinsaft JW , Callister TQ, Min JK . Evaluation of prosthetic valve endocarditis by 64-row multidetector computed tomography . Int J Cardiol $2007 ; 120$ : e27 - e29 . Epub 2007 Jun 27. PubMed PMID: 17597237. 
[16]Tsai IC , Lin YK, Chang Y, et al . Correctness of multi-detector-row computed tomography for diagnosing mechanical prosthetic heart valve disorders using operative findings as a gold standard. Eur Radiol $2009 ; 19: 857$ - 867.

[17]Konen E , Goitein O , Feinberg MS , et al. The role of ECG-gated MDCT in the evaluation of aortic and mitral mechanical valves: initial experience. AJR Am J Roentgenol $2008 ; 191: 26-31$.

[18]Czerwinski R. N., Jones D L. Line and boundary detection in speckle images. IEEE Trans. Image Processing 1998; 7:1700-1714.

[19]Chang-Yan Xiao, Zhang Su, Ya-zhu Chen. A diffusion stick method for speckle suppression in ultrasonic images. Pattern Recognition Letters 2004; (25)16:1867-1877.

[20] González RC, Woods RE. Digital image processing. Prentice Hall. p. 354.

[21]Perona P, Malik J. Scale space and edge detection using anisotropic diffusion. IEEE Transactions on Pattern Analysis and Machine Intelligence, 1990, 12(7): 629-639.

[22]Yezzi A. Modified Curvature Motion for Image Smoothing and Enhancement. IEEE Transaction on Medical Imaging, Vol 7 (3), 1998.

[23] Qian Y, Zhang Y, Morgan M. A comparison of medical image segmentation methods for cerebral aneurysm computational hemodynamics. Biomedical Engineering and Informatics (BMEI), 2011 4th International Conference, 2011, Vol2: 901-904

[24] Brassel M, Bretin E. A modified phase field approximation for mean curvature flow with conservation of the volume, Mathematical Methods in the Applied Sciences Volume 34, Issue 10, pages 1157-1180, 15 July 2011.

[25]Boskamp T, Rinck D, Link F, Kummerlen B, Stamm G, Mildenberger P. New vessel analysis tool for morphometric quantification and visualization of vessels in ct and $\mathrm{mr}$ imaging data sets. Radiographics 2004, 24(1) :287-297. 
[26]William E. Lorensen, Harvey E. Cline: Marching Cubes: A high resolution 3D surface construction algorithm. In: Computer Graphics, Vol. 21, Nr. 4, July 1987.

[27]Bloomfi eld P , Wheatley DJ , Prescott RJ , Miller HC . Twelve-year comparison of a Bjork-Shiley mechanical heart valve with porcine bioprostheses . N Engl J Med 1991 ; $324: 573-579$.

[28]Hammermeister KE, Sethi GK, Henderson WG, Oprian C, Kim T, Rahimtoola S. A comparison of outcomes in men 11 years after heart-valve replacement with a mechanical valve or bioprosthesis. Veterans Affairs Cooperative Study on Valvular Heart Disease . N Engl J Med 1993 ; 328 : 1289 - 1296 .

[29] Puvimanasinghe JP , Steyerberg EW , Takkenberg JJ , et al . Prognosis after aortic valve replacement with a bioprosthesis: predictions based on meta-analysis and microsimulation. Circulation $2001 ; 103: 1535-1541$.

[30]Ruggieri VG, Flecher E, Anselmi A, et al. Long-Term Results of the Carpentier-Edwards Supraannular Aortic Valve Prosthesis. Ann Thorac Surg. 2012 Jul 7.

[31] Piazza N, Bleiziffer S, Brockmann G, et al. D. Transcatheter aortic valve implantation for failing surgical aortic bioprosthetic valve: from concept to clinical application and evaluation (part 2). JACC Cardiovasc Interv. 2011 Jul;4(7):733-42. 


\section{Tables}

Tab. 1: pre-operative echocardiographic assessment and intra-operative failure evaluation of studied bioprostheses.

\begin{tabular}{|c|l|c|l|l|}
\hline Case & Bioprosthesis type & Bioprosthesis Size & Echocardiographic assessment & Failure mechanism \\
\hline 1 - HER & Edwards Perimount & $23 \mathrm{~mm}$ & Aortic regurgitation & Leaflet prolapse \\
2 - MAG & Edwards SAV & $25 \mathrm{~mm}$ & Aortic stenosis & Calcified pannus \\
3 - BOU & Edwards SAV & $23 \mathrm{~mm}$ & Aortic stenosis & Leaflet calcification \\
4 - DAV & Medtronic Mosaic & $23 \mathrm{~mm}$ & Normal & Endocarditis \\
\hline
\end{tabular}

Tab.2: image resolution parameters of in-vivo (IV) and ex-vivo (EV) acquisitions.

\begin{tabular}{|c|c|c|c|}
\hline Patient & Observation & Image size (voxels in $\mathrm{X}, \mathrm{Y}, \mathrm{Z}$ ) & Image sampling (mm in $\mathrm{X}, \mathrm{Y}, \mathrm{Z}$ ) \\
\hline \multirow[t]{2}{*}{ HER } & IV & 512512281 & $0.3125 \quad 0.3125 \quad 0.625$ \\
\hline & $\mathrm{EV}$ & 512512198 & $\begin{array}{lll}0.3125 & 0.3125 & 0.3125\end{array}$ \\
\hline \multirow[t]{2}{*}{$M A G$} & IV & 512512105 & $\begin{array}{llll}0.318 & 0.318 & 0.312\end{array}$ \\
\hline & $\mathrm{EV}$ & 512512110 & $\begin{array}{lll}0.0966 & 0.0977 & 0.312\end{array}$ \\
\hline \multirow[t]{2}{*}{ BOU } & IV & 512512153 & $\begin{array}{llll}0.3632 & 0.3632 & 0.625\end{array}$ \\
\hline & $\mathrm{EV}$ & 512512120 & $\begin{array}{lll}0.0977 & 0.0977 & 0.312\end{array}$ \\
\hline \multirow[t]{2}{*}{ DAV } & IV & 512512290 & $\begin{array}{llll}0.418 & 0.418 & 0.635\end{array}$ \\
\hline & $\mathrm{EV}$ & 512512125 & $\begin{array}{llll}0.0977 & 0.0977 & 0.3125\end{array}$ \\
\hline
\end{tabular}




\section{Figures Legend}

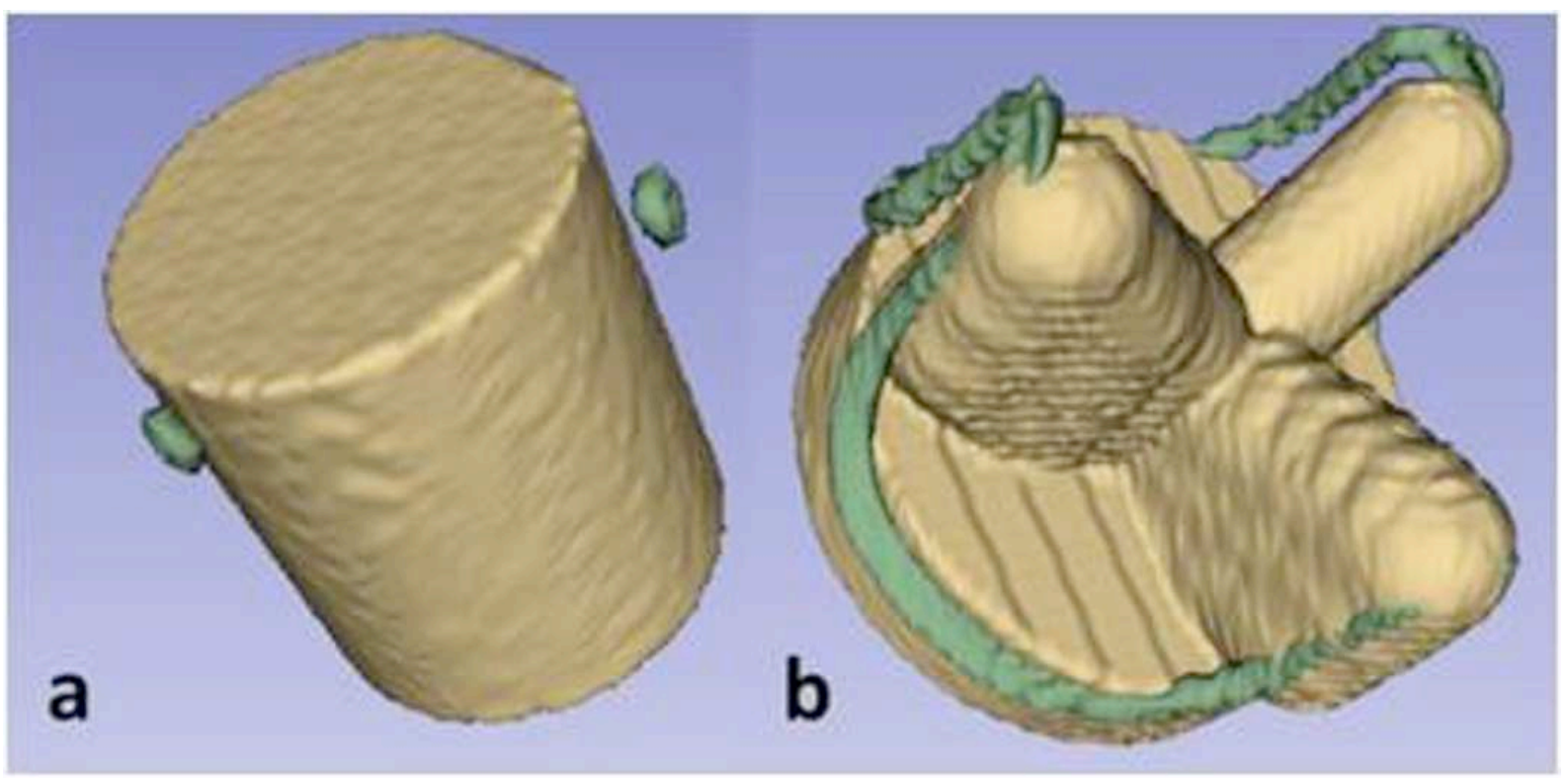

Fig. 1: 3D representation of a cylindrical (a) or conformational (b) region of interest.

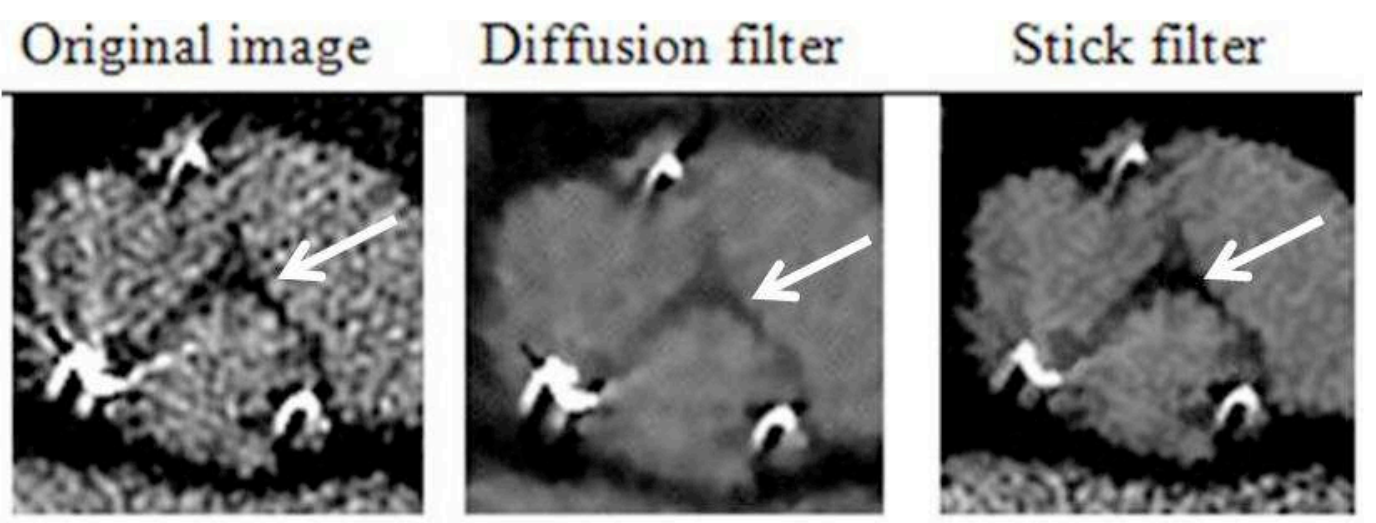

Fig. 2: Example of results about pre-processing by diffusion and stick filters for case n 3 BOU. The same section, before and after filtering processes, is presented including the stent (bright) and bioprosthetic leaflets (dark). Leaflets are indicated by white arrows. 


\section{SNR}

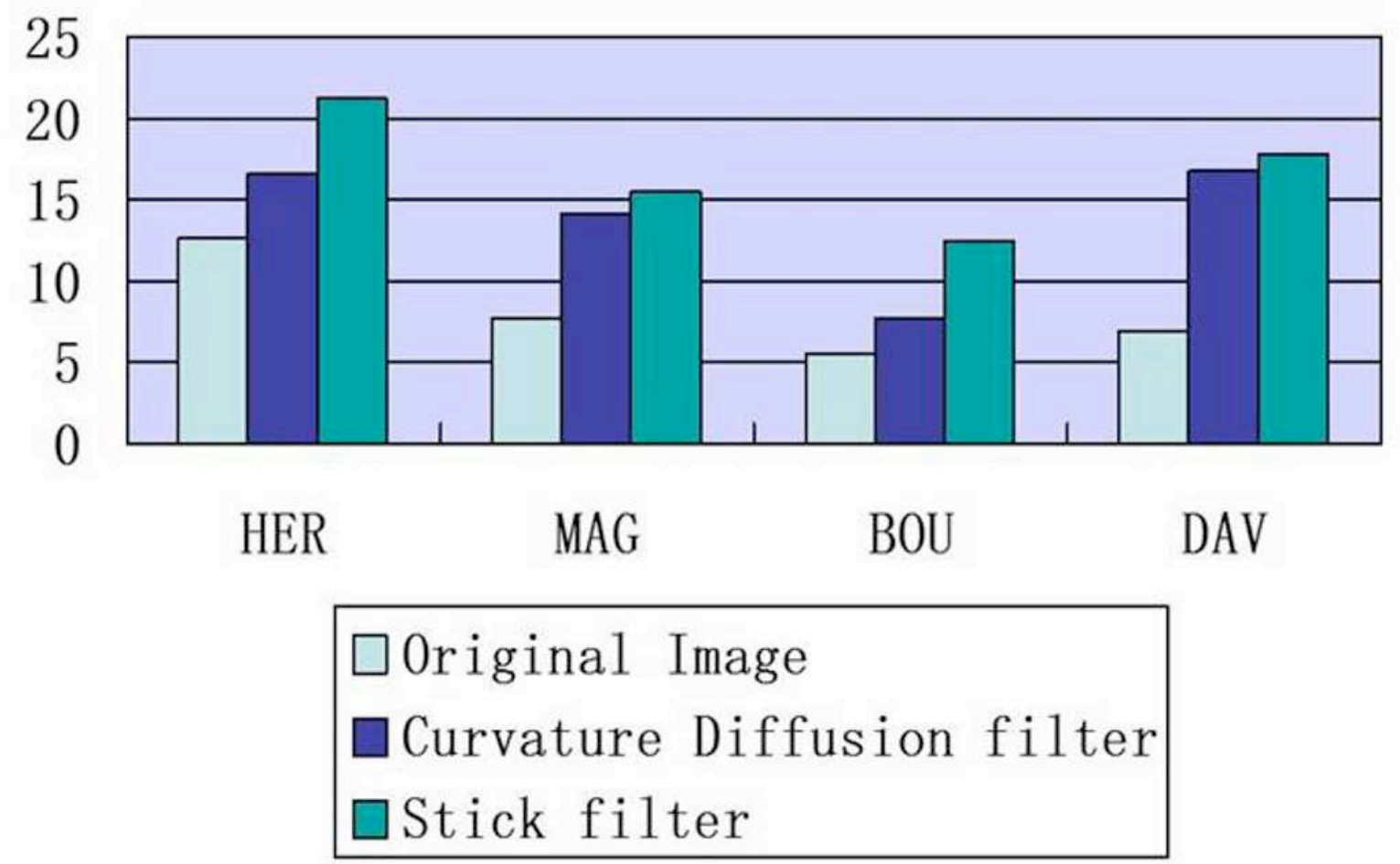

Fig. 3: Results of preprocessing (SNR) with stick filter and curvature diffusion filter for each database. 


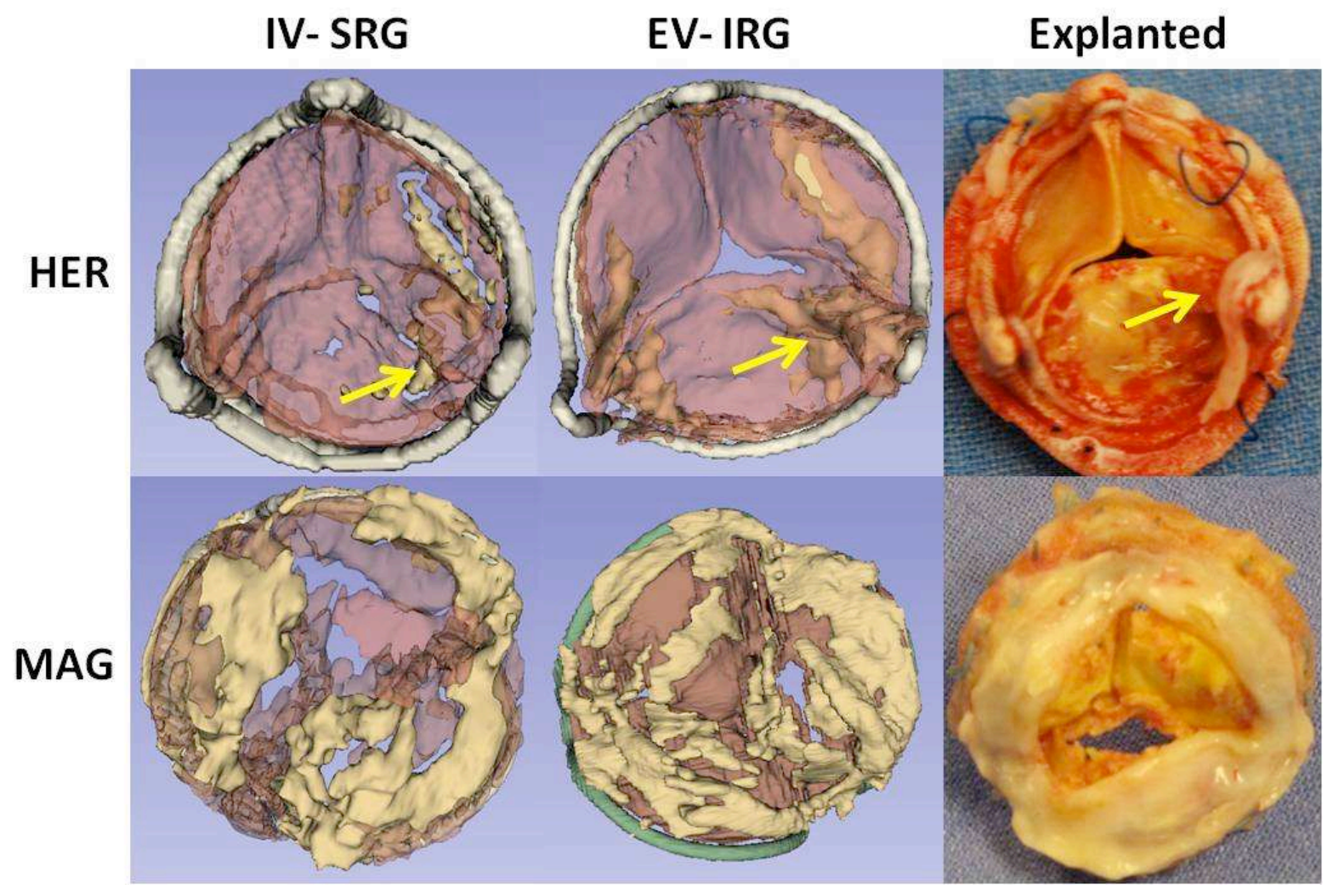

Fig. 4: Examples of segmentation results for cases HER (the yellow arrows show the commissure lost because of leaflet prolapse) and MAG (the calcified pannus is well detected). 


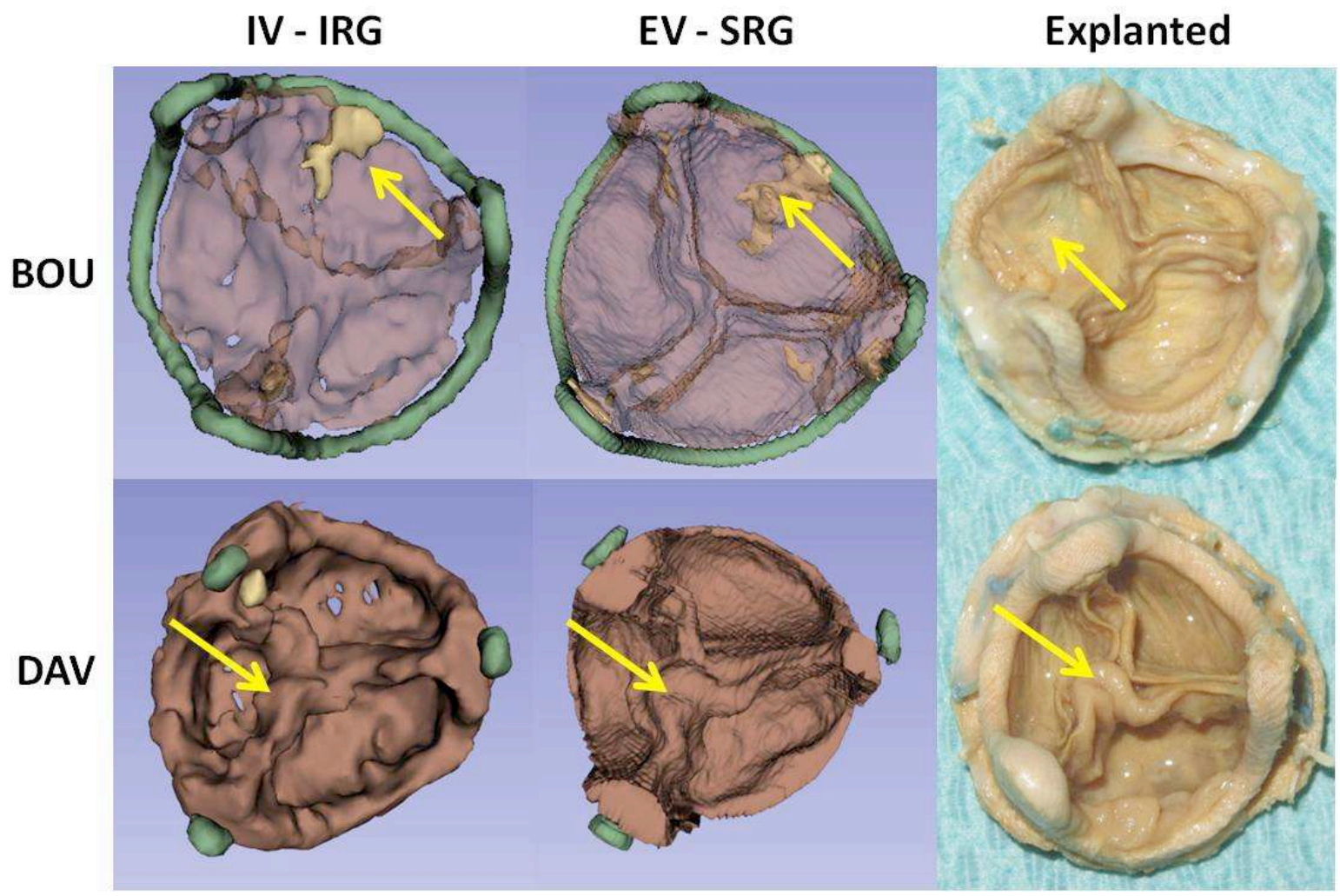

Fig. 5: Examples of segmentation results for cases BOU (yellow arrows show the calcified leaflet) and DAV (yellow arrows indicate leaflet thickening). 

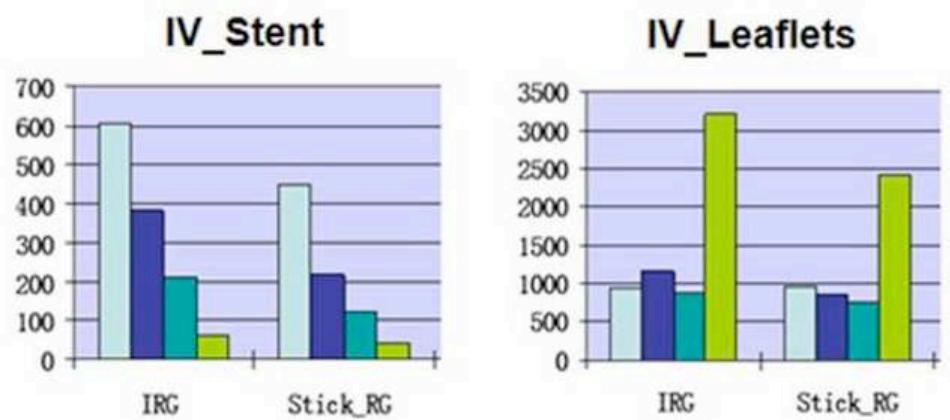

IV_Calcif/Pannus

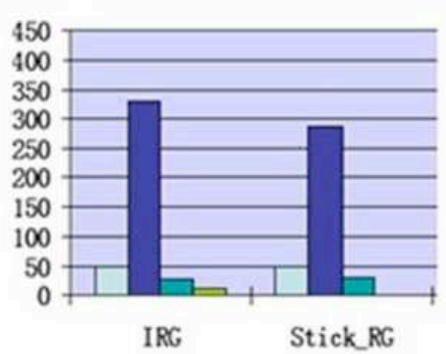

EV_Stent

EV_Leaflets
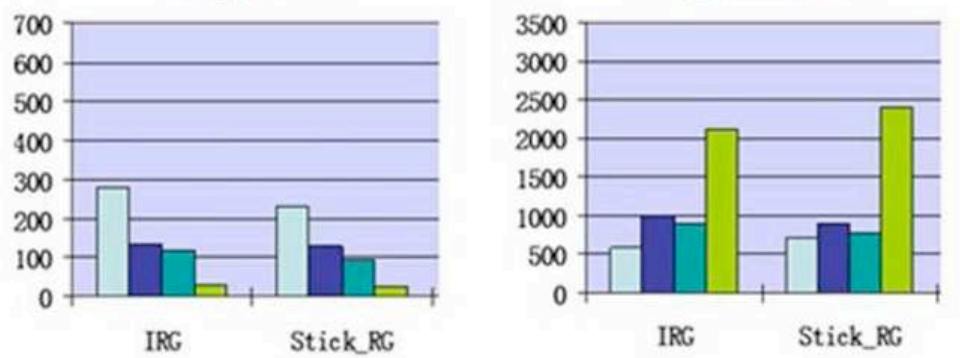

EV_Calcif/Pannus

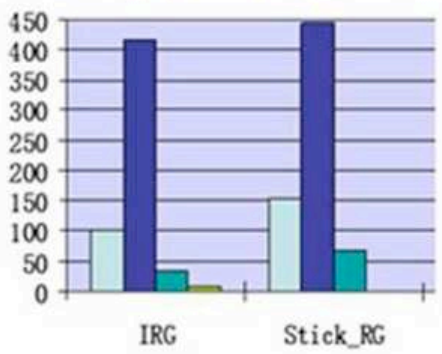

$\square$ HER $\square$ MEG $\square$ BOU $\square$ DAV

Fig. 6: Volume analysis of bioprosthesis components (volumes expressed in $\mathrm{mm}^{3}$ ). 